Medicine Updates Faculty of medicine April 2022,volume 9, issue 9 https://muj.journals.ekb.egdean@med.psu.edu.eg vice_dean_postgraduate@med.psu.edu.eg DOI: 10.21608/muj.2021.101396.1076

ISSN : 2682-2741

\title{
" Medications errors prevention and its role in patient safety management"
}

\section{Authors}

Alaa Mohamed 目 ${ }^{1}$; Fatma Hussin El adawy ${ }^{1}$; Lina Mohsen

Mahfouz $^{1}$; Amr Elsayed Elshamy²; Mennatallah Mostafa Ibrahim ${ }^{3}$; Heba Elsayed $^{4}$; Ahmed Abdelmohsen Abdelmotaleb ${ }^{5}$

${ }^{1}$ Faculty of Pharmacy

${ }^{2}$ Faculty in oral and Dental Medicine

${ }^{3}$ Faculty of pharmacy Sinai University

${ }^{4}$ Portsaid University

${ }^{5}$ College of health sciences 
Abstract:

\section{Background:}

Patients are at high risk for injury or death every day because of medication errors (1).

Organized and systematic tactics to improve tasks, technology, environment and organizational culture associated with medication system are required for patient safety.

Method: we review medication error reporting system in last three months in general ward of hospital X.

Conclusion: Reporting system and reviewing and correcting causes that leads to ME may help to prevent ME, specially that reach patient. (Near miss)

It reveals that incidence of ME is decreased and especially ME that cause patient harm.

\section{Recommendations:}

Within hospital, areas of medication ordering, dispensing, administrative should be well known (5).

of medication About Possibility that might go wrong during utilization administration devices (e.g. infusion pumps) should be well known by nurse (6). Pharmacists should self-check or double check with other pharmacist in reading prescriptions, labeling and dosage calculations (7).

\section{Key Words:}

Medication Errors, Medical Errors, prevention, patient safety.

\section{Introduction:}

Medication error is "any preventable event that may result in the use of an inappropriate medication in harm to a patient while the medication is under the control of the healthcare professional, patient, or consumer," according to the 
National Coordinating Council for Medication Error Reporting and Prevention (8).

Medical errors are the 3rd major source of death in the United States, after heart illness and cancer (9).

Medication errors are more likely to happen in elder people, especially in the presence of Co-morbidity and Polypharmacy (11).

\section{Methodology:}

- We conduct a reporting system as a google form and paper form available to all healthcare providers in hospital X.

- Training all healthcare providers on ME definition, causes and how to report it.

- Up on reviewing the reporting system, and correcting causes that leads to medication error, it helps to decrease incidence of ME.

\section{Result:}

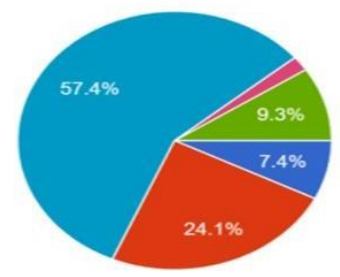

\section{Causes:}




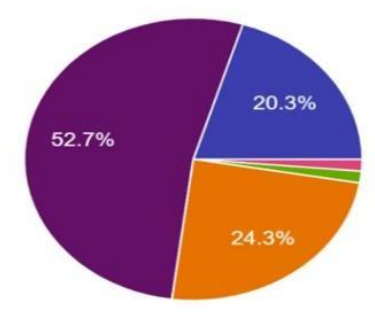

Mishandled Verbal Order

Sound alike medication

- Look alike medication

Confusing product labeling

- Confusing drug insert

Incomplete patient information

- Misleading advertisement

Unclear/Lacking documentation.

A $1 / 3 \mathbf{\nabla}$

Severity level:

\begin{tabular}{|l|l|}
\hline Query2 & $\begin{array}{l}\text { co } \\
\text { unt }\end{array}$ \\
\hline Severity level & 7 \\
\hline $\begin{array}{l}\text { B mistake that did not } \\
\text { arrive the patient }\end{array}$ & 113 \\
\hline $\begin{array}{l}\text { C mistake that arrived } \\
\text { patient but out of the way } \\
\text { to cause damage }\end{array}$ & 17 \\
\hline $\begin{array}{l}\text { D mistake that arrived the } \\
\text { patient and could have } \\
\text { required controlling }\end{array}$ & 2 \\
\hline $\begin{array}{l}\text { E mistake that could have } \\
\text { caused temporary harm }\end{array}$ & \\
\hline
\end{tabular}

Medication mistake included:

Prescribing drug

Dispensing drug

Administration of drug

\section{Prescribing drug:}

Improper Drug-chosen for a patient.

Incorrect dose volume of drug.

Weakness Responsible practitioners as physician

Contributing factors:

Handwriting is not understood.

Drug pedigree unclear.

Equivocation with medicine name. 
Employ of abbreviations.

\begin{tabular}{|l|l|l|}
\hline shortcuts & Why it's an issue & Stand by \\
\hline collective & $\begin{array}{l}\text { Erring for zero } \\
\text { Number four or cc }\end{array}$ & Record unit \\
\hline IU & Erring for IV or number ten & Record internation \\
\hline QD & Erring for QID & Record daily \\
\hline QOD & Erring for QID and QD & Record every other \\
\hline Trailing zero & Miss the decimal point & Record $\mathrm{xmg}$ \\
\hline Lack of leading zero & Miss the decimal point & Record o.x mg \\
\hline
\end{tabular}

\section{Use of Verbal order.}

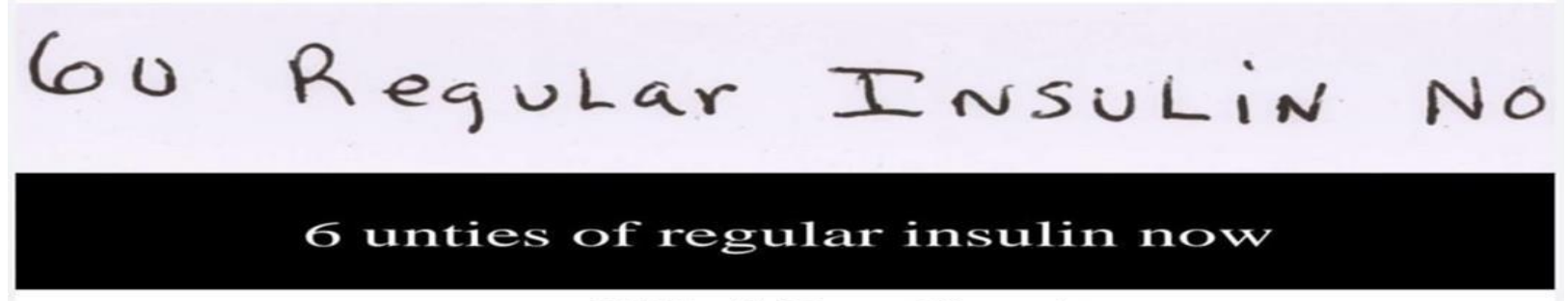

Filled Rx: 60 units

\section{Prescribing:}

Electronic prescribing may help to reduce mistakes resulting from impenetrable hand line.

Computerized physician order entry system (CPOES), eject the need for reproduction of orders by nursing.

\section{- Dispensing:}

Occurs at the interval between receiving the prescription in pharmacy and giving the dispensed medications to the patient.

\section{- Contributing factors:}

- Substituting one medication with another similar one.

- Drugs that look a-like or sound a-like are not separately placed. 
- Distractions and interruptions.

- Dispensing medications through verbal order without seeing the written one.

- Weak knowledge about new medications.

- The references used are outdated or containing un-reliable information

\section{- Dispensing :}

- Be aware when dispensing medications that look a-like or sound a-like.

- Be aware when dispensing High alert medications (HAM).

ex. *Hypertonic electrolytes (potassium chloride, calcium chloride, magnesium sulphate)

*Cytotoxic agents

*Insulin

- Keep the work area calm and organized to reduce stress.

- Avoid interruptions as possible.

\section{Administration:}

Inconsistency between the drug therapy received by the patient and drug therapy written by the prescriber. That may be .....

1) Errors of distraction _ drug not given

2) Improper administration technique or drug

3) Negotiate lack of commitment in guidelines

Responsible practitioners: nurses

\section{Examples of medication administration errors:}

- additional dose mistake

- improper dose mistake

- improper route mistake 
- improper rate mistake

- improper potion form

- improper time mistake

- improper management mechanics

- management of wrong preparation

- Distraction error (ex. Lack of stock).

- Having the imposition, medication and patient in same site to be restricted versus one another.

- Distinct checking for dosage calculation by another healthcare professional before drug administers.

- Minimizing interruptions during drug rounds.

- Look out of look-a-like \& sound-a-like drugs.

- Regulate the workplace.

- Minimize distraction.

- Being aware of High Alert Medications (HAM), drugs that have a risk of causing considerable patient damage when used in mistake.

Plain strategies to avert drug mistakes:

\section{Patient information}

- His full name, his height and weight.

- His history to verify any allergics or reactions to give him accurate diagnosis.

\section{Drug information:}

Using reference: guidelines and high alert medications.

\section{Communication:}

- Improve hand writing to avoid any abbreviations and be aware of soundlike drug. 


\section{Labeling and storage:}

Storage area well organized.

- Store medication alphabetically.

- Separate problematic drugs \& disconnect outer solutions, non-drug elements, workout solutions.

- Control temp. \& expired date.

- Staging should be at eye level with attachments return forward.

- Control access to medications.

\section{Drug devices:}

Proper drug devices (right syringes gauge, etc..

Train staff to use the system completely.

\section{Patient education}

Evaluate patient's medical literacy \& tailor the information

Do not rush medication counseling, asked to reiterate back the information to ensure patient perception.

Ask the patient about ability to pay for medication or follow instructions as Non-compliance is VIP for Omission.

\section{7) Culture change}

Assess your practices performance authorize all team members to arrow information widely and freely.

Create it simple to learn from mistakes, as mistakes will be looked odds for instructions, not penalization.

Look for system modifications that will help block future mistakes. 


\section{Conclusion:}

Reporting system and reviewing and correcting causes that leads to ME may help to prevent ME, specially that reach patient. (Near miss).

\section{The following recommendations to minimize medication error:}

1. Using the principles of the formulary system, the P\&T board should be responsible for formulating policies and list of medications to be supplied.

2. Jealousy and discussion must be given in leasing and appointing personnel.

3. Adequate personnel must be ready to do actions enough, workload must be assessed.

4. Appropriate business ambience must occur for the production of drug products.

5. Potential mistake heads during the business ambience, such as recurrent interruptions must be specified \& reduced.

6. Areas of medication ordering, dispensing $\&$ administration during hospital must be obviously qualified.

7. Hospital system should include sufficient written and oral communication among health care professional, modifications desired to emend improper instructions must be regarded as prospect mistakes.

8. There should be quality program enclose safe use of medicine, drug use appraisal program should be advanced (pharmacy / nurse/quality), keep an eye on suitable hire of drugs linked with peak hesitation of ADR, antimicrobials, chemotherapy, CV drugs, injectable dosage form, check and account medication error to identify causes of error.

9. Clinical pharmacists should have routine access to patient information to evaluate the suitability of medication order.

10.registration of patient medication profiles (recording and intercourse of patient's current medications and emptying medication). The organization implements a process to gain and record a full roll of the patient's influx 
drugs onto approval to the community \& together the embroilment of the patient.

11.procurement, allocation, \& monitoring of all drugs used within the organization must be responsible by The pharmacy department. 24-hour pharmaceutical service is strongly recommended in hospital.

12.policies that supply effective and safe allocation of all medication should developed by Pharmacy director, P\&T committee and department of nursing should develop.

13.For non-necessity medication, from the pharmacy department for individual patients whole futile and non-futile drug should be divided.

14.Reduce the stock piling of nen emergency floor paper drugs.

15.Drug with tight therapeutic index e.g. digoxin and concentrated forms should be routinely scanned to enclose appropriate packaging, labeling , storage .

16.External use drugs are stocked separately from internal use drugs.

17.The pharmacy manager and staff must include that all drugs in hospital of good quality and impartiality (bioavailability, enough drug packaging and labeling, expiry date ).

\section{2- Recommendations for prescribers:}

- It was estimated that $1 \%$ of the patients in the patients had reverse events as a result of poor medical management, reading starts:

- Before prescribing additional drugs. suitable treatment of signs and symptoms

- Hospitalization, use of medications (e.g. prescriptions, medication times, confirmed abbreviations).

- Medications should be medication requests (including, including patient, generic drug name, brand name) and direction time if needed. 


\section{3- Recommendations for pharmacists:}

- Perfectly, pharmacist will cooperate with the descriptor in development of treatment planning to output specific treatment outcomes for the patient. Recommendations

- Check of the computation and effect rates and other calculations by the clinical pharmacist.

- To admonish and identify suitable drug therapy, the clinical pharmacist should be ordinary with the urbanity and check out with colleagues and other healthcare professionals in continuing professional education programs.

- Pharmacists must be customary for medication continuing planning system, The policies and procedures of drug allocation to should be pure, comprehend by pharmacist, medical staffs and nursing staffs.

- Medication orders should not guess the intent of embarrassing by dispensing pharmacists. If there are any problems, before dispensing the prescriber should be contacted.

\section{4-Recommendations for nurses:}

Nurses in a beautiful dream mode.

The following argument is displayed on the medication:

1) The nurses must know the medication ordering system.

2) nurses must carefully dispense the medicines before giving the first commission and compare them with the medicines that were dispensed on them.

3) Copying orders should be avoided and recognized as initial opportunities for errors. 
- 4) The doses of medications that were dispensed before they were given. The procedure in the pharmacy department is the appropriate procedure.

- 5) Verify the patient's identity before giving each prescribed dose.

\section{Recommendations for patients and personal caregivers:}

1) Patients (or their caregivers or their representatives) have the right to know all aspects of their care, including drug treatment.

2) Primary care is provided for care, the emergence of patients using medication. 3) Patients should learn the names of the medicinal products to be prescribed and administered, and the strengths and dosing schedules.

Patients keep a personal list of all drug treatments (prescription drugs, prescription drugs, home remedies, and medicinal foods).

4) You should also use the medicines contained in the medicines. Share this status.

5) Providing medical advice, medicines, medicines, medicines, medicines, medicines on demand.

\section{References:}

1. Wittich CM, Burkle CM, Lanier WL. Medication errors: an overview for clinicians. Mayo Clin Proc. 2014 Aug;89(8):1116-25. [PubMed]Whittaker CF, Miklich MA, Patel RS, Fink JC. Medication Safety Principles and Practice in CKD. Clin J Am Soc Nephrol. 2018 Nov 07;13(11):17381746. [PMC free article] [PubMed]

2. Ibrahim OM, Ibrahim RM, Meslamani AZA, Mazrouei NA. Dispensing errors in community pharmacies in the United Arab Emirates: investigating incidence, types, severity, and causes. Pharm Pract (Granada). 2020 Oct-Dec;18(4):2111. [PMC free article] [PubMed] 
3. Zirpe KG, Seta B, Gholap S, Aurangabadi K, Gurav SK, Deshmukh AM, Wankhede P, Suryawanshi P, Vasanth S, Kurian M, Philip E, Jagtap N, Pandit E. Incidence of Medication Error in Critical Care Unit of a Tertiary Care Hospital: Where Do We Stand? Indian J Crit Care Med. 2020 Sep;24(9):799-803. [PMC free article] [PubMed]

4. Sim MA, Ti LK, Mujumdar S, Chew STH, Penanueva DJB, Kumar BM, Ang SBL. Sustaining the Gains: A 7-Year Follow-Through of a HospitalWide Patient Safety Improvement Project on Hospital-Wide Adverse Event Outcomes and Patient Safety Culture. J Patient Saf. 2020 May 09; [PubMed]

5. Aseeri M, Banasser G, Baduhduh O, Baksh S, Ghalibi N. Evaluation of Medication Error Incident Reports at a Tertiary Care Hospital. Pharmacy (Basel). 2020 Apr 19;8(2) [PMC free article] [PubMed].

6. Neal JM, Neal EJ, Weinberg GL. American Society of Regional Anesthesia and Pain Medicine Local Anesthetic Systemic Toxicity checklist: 2020 version. Reg Anesth Pain Med. 2021 Jan;46(1):8182. [PubMed]

7. Kefale B, Degu A, Tegegne GT. Medication-related problems and adverse drug reactions in Ethiopia: A systematic review. Pharmacol Res Perspect. 2020 Oct;8(5):e00641. [PMC free article] [PubMed]

8. Gonzaga de Andrade Santos TN, Mendonça da Cruz Macieira G, Cardoso Sodré Alves BM, Onozato T, Cunha Cardoso G, Ferreira Nascimento MT, Saquete Martins-Filho PR, Pereira de Lyra D, Oliveira Filho AD.

Prevalence of clinically manifested drug interactions in hospitalized patients: A systematic review and meta-analysis. PLoS

One. 2020;15(7):e0235353. [PMC free article] [PubMed]

9. Uhlenhopp DJ, Aguilar O, Dai D, Ghosh A, Shaw M, Mitra C. HospitalWide Medication Reconciliation Program: Error Identification, CostEffectiveness, and Detecting High-Risk Individuals on Admission. Integr Pharm Res Pract. 2020;9:195-203. [PMC free article] (PubMed) 